\title{
Effectiveness of the Service Mapping Method in Re-designing the Bariatric Surgery Clinical Pathway of a Public Hospital
}

\author{
Loreggian Lara $^{1}$, Sartor Maria Elisa ${ }^{2}$, Strohmenger Laura ${ }^{3}$ and Rovati Marco Pietro Lucca ${ }^{4}$ \\ 1. The Department of Medicine of St Paul Hospital, Milan University, Milan 20142, Italy \\ 2. The Department of Economics, Management and Quantitative Methods, Milan University, Milan 20122, Italy \\ 3. The Department of Biomedical Surgical and Dental Sciences of St Paul Hospital, Milan University, Milan 20142, Italy \\ 4. The Department of Surgery of St Paul Hospital, Milan University, Milan 20142, Italy
}

\begin{abstract}
Background: Nowadays obesity is the world's common disease. Bariatric surgery is the only therapy that provides significant cost savings within Public Health Service, but the lack of diagnostic paths universally accepted causes enormous waste of resources and disruptions. Service Mapping is the ideal methodology to describe work's organization and to plan a new service model. Methods: The Service Mapping has been used to represent the actual state of the bariatric surgery service and starting from the critical aspects found, we have developed a desirable state of the service. Results: Experience-based design has given centrality to the beneficiary, making the bariatric service sensitive to patient's needs and expectations. The micro-organization of work has improved professionals' integration, avoiding the creation of new operational entities or additional costs. The service has been simplified both for clinicians and hospital managers. The strategic repositioning of the dietician and general practitioner's recognition within the bariatric path allowed us to achieve better clinical outcomes. Conclusions: Service Mapping has highlighted clinicians’ difficulties in providing the service, emphasizing the importance of the beneficiary. The iconic representation is a powerful explicit framework, fundamental for management purposes, to understand the role of every subject involved in the service, to rationalize work's organization, and integrate healthcare activities.
\end{abstract}

Key words: Service mapping, bariatric surgery, obesity, public health costs, national health service, management.

\section{Introduction}

\subsection{Background}

Obesity has reached the dimensions of a real global epidemic, called "globesity" [1]. Currently, almost half of the world's population is overweight, while the percentage of obese people ranges from $10 \%$ to $20 \%$ for men and from $15 \%$ to $25 \%$ for women [2]. In children and adolescents, obesity accounts respectively 160 and 40 million of diagnosed cases and, according to experts' opinion, these numbers are expected to increase.

Obesity absorbs almost 8 billion euro per year, about $7 \%$ of the global health spending [3]. Scientific studies demonstrate that at each additional unit of
$\mathrm{BMI}^{1}$ corresponds an increase of $2.3 \%$ on healthcare costs, and no other disease affects so heavily on the economic balance of a State [4]. Type II diabetes, dyslipidemia, hypertension, vasculopathy, cardiopathy, pneumopathy, joint syndromes and many tumours are obesity-related pathologies that significantly increase the mortality of obese patients [5]. Obesity reduces the life expectancy of at least 9 years in women and 12 years in men [6]: in EU about 1 of 13 deaths per year is attributable to weight excess [7]. In Italy there are 16.5 million of overweight people and 5.5 million obese

\footnotetext{
${ }^{1}$ BMI (Body Mass Index) is a biometric data, expressed as the ratio between weight $(\mathrm{kg})$ and the square of the height $(\mathrm{m})$, used in an epidemiological context to define the physical state of a subject (thinness, normal weight and the various levels of obesity).
} 
subjects, and the health care costs for the cure of these patients are extremely high [8]:

- 2,700 $€$ spent for a patient without serious complications

- $3,900 €$ if the subject is diabetic

- $6,500 €$ if pneumopathic

- 5,500 € if hypertensive

- $12,000 €$ if the patient has osteoarticular diseases and requires a prosthesis intervention

- 7,200 $€$ if he needs dietotherapy and food supplementation

Obesity-related health care costs are around 23 billion euro annually, of which 11 billion totally supported by the National Health Service (over 60\% for hospitalizations, more than $10 \%$ for diagnostic tests and about $15 \%$ for drugs and specialist examinations) [8]. It is important to emphasize that in obese subjects, pathologies adds up: $26 \%$ of obese people suffer from diabetes, 23\% from asthma, 44\% from arthritis, 51\% from hypertension and 52\% from various forms of cancer[9, 10]. That's why today's amount of indirect costs (such as premature deaths, reduction of productivity, absence from work, pension subsidies, unemployment, etc.) is around 65 billion euro per year. Facilitating the access of obese patients within the appropriate surgical paths we could reduce mortality and obesity, improving the quality of life of these people [11] and reducing the tax burdens on Public Health [12].

\subsection{Costs and Benefits of Bariatric Surgery in the} Treatment of Pathological Obesity

Bariatric surgery was developed in the 1950s, especially for the treatment of pathological obesity. Every year, surgeons perform about ten thousand of bariatric interventions and clinicians confirms that investing on surgical therapy there would be benefits for both patients and for the budget of the National Health Service [13]. Although 3\% of the obese population is eligible for bariatric surgery, data show that only $0.8 \%$ of these patients access to surgical therapy; an irresistible percentage if we consider that the population excluded from bariatric surgery costs at least $€ 8$ billion annually to Public Health Service [14].

The mortality rate of bariatric surgery-calculated on the world's largest register, which counts more than 42,000 patients-is just $0.17 \%$ and enjoys a success rate of $98 \%$ [15], against a failure rate of the dietetic approach which is close to $95 \%$ [16]. While researchers aim to divulge these outstanding medical achievements, they also keen to point out that surgery is not a shortcut to cure obesity.

In Italy, no epidemiological studies have been published on a national scale yet, but a prospective follow-up simulation (at five years) has shown the amount of cost savings due to bariatric surgery:

- About-49,770,000 € for the treatment of type II diabetes

- $129,400,000 €$ in regard to hypertension

- $15,750,000 €$ to cure of coronary heart disease

- $14,906,000 €$ in relation to dyslipidemia

- 119,616,000 € to treat arthrosis

- 65,100,000 $€$ for the clinical management of respiratory distress

Against an initial expenditure of $€ 235$ million due to the surgical treatment, Public Health would save about $€ 394,454,000$ [17], with a reduction of mortality around 60-93\%, results extendible to all bariatric surgery centres. These studies show that bariatric surgery improves the quality and the amount of life of obese people and allows a reduction of the social and health costs that no other modern therapeutic approach can offer.

\section{Objective}

The present research is a management intervention study concerning the bariatric surgery service of an important public hospital of Milan. Because of an alarming epidemiological situation, and in light of the concrete benefits of bariatric surgery in the treatment of obesity, the project aims to plan a more efficient clinical pathway and to adopt a new diagnostic protocol. 
The primary objectives were:

- Return the centrality to the beneficiary (meet patient's needs and expectations)

- Design the service in the experience-based perspective (co-designing it with the patient)

- Improve the micro-organization of work

- Integrate clinical and therapeutic practices with management activities

- Temporally and spatially relocate the clinical activities without extra costs

- Simplify the service for both the beneficiary and the providers (clinicians and managers)

- Give centrality to the direct communication between professionals involved

- Relocate the dietician's professional figure

- Recognize the role of the general practitioner within the bariatric surgery service

- Make the service efficient (economically advantageous for top management and NHS)

The objectives mentioned above have been used as design criteria, in order to implement the bariatric surgery service of the hospital.

\section{Methods}

\subsection{The Service Mapping as Analytical and Design} Tool

The Service Mapping is the most useful methodology to analyze economic and territorial contexts, to evaluate the organization of companies, plans and business projects [18], a versatile management tool also applicable to Public Health. Service Mapping allows to represent (and understand) the logics behind the decision-making and all activities occurring within clinical settings. It is a complex process-based on design activities (iconic representation) and modelling (adaptation of the service's elements) —used to gather information, to rationalize, and expose them in a suitable way for critical analysis.

The service representation-called "service map"-is a powerful analytical tool, whose iconic results are useful to design a new delivery system. The map highlights the recipients of the service and the organizational, management or communicative implications of the service provided. Thus, all activities related to the service will be studied and programmed.

The process is drawn from left to right as a series of actions in chronological order, while the structure of the service is located on the vertical axis, as the levels of an overturned organizational chart [19]. The beneficiary's actions are placed at the top of the map, followed in parallel by the service provider's activities, and divided from them by the "interaction line". The activities of the provider are classified as front-line and back-line actions, separated from the "visibility line". The "internal interactions line" separates support functions from administrative ones, dividing the ways in which the service is delivered from the responsibilities for its management.

Within the map, the significant places of the service can be aggregated into four main areas:

- Front-line. Contact point between the beneficiary and the health care operators. In this area doctors, nurses, and employees of the CUP ${ }^{2}$ provide the sub-services of their own competence to the beneficiary.

- Back-line. Space beyond the front-line area, invisible to the beneficiary. In this place head nurses, departments' chiefs and medical directors fulfil all tasks indispensable for the provision of sub-services, but not delegable to the front-line professionals.

- Area of internal relationships. Place that includes mere organizational activities, where only the interactions between back-line operators occur (Acceptance and Reception Office, Pharmacy, Economical Superintendency).

- Support area. Zone of the service where operational units or medical directors perform managerial tasks to support back-line professionals (Strategic Direction and SITRA ${ }^{3}$ ).

\footnotetext{
2 Centro Unico Prenotazioni: Unified Reservation Center (English).

3 Servizio Infermieristico Tecnico Riabilitativo: Technical Rehabilitation Nursing Service (English).
} 
The service must be represented in all its components, inside and outside the healthcare facility considered. The communicative acts may be physical or visual, face to face or device-mediated (telephone, e-mail, fax, etc.). So, the analyst becomes aware of the resources asked to the beneficiary to access the service, how he participates in its delivery, if he has an appropriate "use-participation culture" (which allow him to live a positive experience), whether the beneficiary is facilitated to earn a sufficient culture of use-participation of the service [20,21] or if the service really has the features of the "service" itself: does the service totally shifts the onerous business/delivery aspects on the suppliers? Does it relieve the workload of the beneficiary? If not, it would be necessary to reorganize the whole service, or at least a part of it [22]. The beneficiary represents far more than a patient: is an unrepeatable individual who experiences, within the service, an unrepeatable life experience [23].

The Service Mapping enhances the quality of services to which it is applied and allows to achieve important strategic goals:

- Analysis and organizational design

- Draw a map that describes the delivery system (current status map)

- Critically consider the kind of interactions and communication channels among operators

- Focus on specific aspects of the service provided (beneficiary and/or sub-services)

- Plan and iconically represent a new service (desirable state map)

- Innovate the services, incrementally or radically

- Support the change-management process

In light of that, the solutions proposed will be coherent with the context, because it is drawn on the basis of concrete objectives and realistic development opportunities [24, 25].

\subsection{Analysis Tools alongside the Service Mapping}

- Documentary analysis

It requires the examination of the Regional Health
Service's macro-structure, the hospital's organizational chart, customer satisfaction reports, the Triennial Performance Plan and the P.O.A. ${ }^{4}$, as well as the minutes of meetings, official acts and patients' health records. Each document is essential to understand hospitals' functioning and to identify the services that need to be redesigned.

\section{- Customer satisfaction}

Source of information on patients' experience that identifies the weaknesses of the service. The reports analyzed include the department of Medicine, Surgery, Gastroenterology, Neurology, Cardiology, Radiology, Gynaecology and Obstetrics, Rehabilitation and Pneumology, all operating units that perform clinical activities in the bariatric surgery service.

- Field observation

Data collection strategy, useful to confirm information from document sources and to highlight the difficulties of operators during service delivery. Field observation is carried out in real (daily) life situations, where the behaviour of individuals springs spontaneously. The researcher remains outside the context, in order to include in the maps all the elements of the environment observed. The analyst thus maintains a continuous control on his tasks and on the others; he acquires the fair degree of separation even when personally involved in the dual role of clinician and programmer.

- Interview with beneficiary and healthcare staff

The expected quality, compared to perceived one, determines the satisfaction (or dissatisfaction) of the beneficiary and depends on: the social context of who judge, his knowledge about the service, the trust on the system and on professionals working in it, the quality of the past experiences, etc. Interviews reveal the socio-demographic characteristics of respondents, their state of health at the time of delivery, the length of stay - or length of work, if employees - within the service and all variables related to it. All information

\footnotetext{
${ }^{4}$ Piano di Organizzazione Aziendale: Business Organization Plan (English).
} 
obtained is important to highlight the interaction points and the gaps between $\mathrm{ATS}^{5}$ and hospital, in order to formulate improvement hypotheses.

\section{Results}

\subsection{The Current Delivery System of the Bariatric Surgery Service}

In the current state map of bariatric surgery service (Fig. 1-scatter service map ${ }^{6}$ ) the user is no more considered as a "customer" but as a "patient-beneficiary", thus gaining a privileged role within the healthcare pathway. Illustration and qualification of individuals' behaviours are discriminated into two forms of interaction (direct and device-mediated). Face to face contacts are represented by black lines, whereas device-mediated interactions are depicted by orange one. Iconic representation highlights the patient's actions, his role in each phase of delivery, the requests he must satisfy during the process and the resources used by the team members, with great attention for the number and type of interactions.

\subsection{Criticality Detected}

\subsubsection{Informational asymmetry}

Any obese subject who wishes to undergo bariatric surgery intervention can't verify if the hospital provides this service and what type of intervention. Even psychiatric suitability to surgery is never mentioned. In fact, many patients judged psychologically unsuitable for for surgery abandon the intention to cure themselves once doctors preclude the surgical options and explain them the standard therapy (an integrated program of diet, physical activity and drugs).

\footnotetext{
${ }^{5}$ Agenzia di Tutela della Salute: Health Protection Agency (English).

6 The title "Scatter Service" refers to the actual state map, as a result of the conceptualization of the criticality detected: many isolated front-line episodes (clinical examinations) and hyper fragmentation of diagnostic activities disorientate the patient and weaken the system by dispersing resources and value within it.
}

4.2.2 Difficult access to the public bariatric path

Normally the general practitioner doesn't diagnose obesity, preventing patients' access to the Public Health service network. Sometimes, instead, the general practitioner, once diagnosed the patient's obesity does not submit the subject to the National Health Service's clinics; without a prescription, the general practitioner ask the patient to undergo medical examinations, putting to his "common sense" the blame to request these medical services.. The role of the general practitioner in the obese patient's diagnostic pathway is completely irrelevant in every aspect, both clinical and operational (Fig. 2).

\subsubsection{Long and expensive diagnosis pathway}

The current state map (Fig. 1) makes it clear how fragmented is the service provided to the patient. The number of diagnostic examinations disperses financial resources of the healthcare system and patients' one. A subject candidate to bariatric surgery has to bear high costs before surgery: Table 1 shows a comparison between NHS (national health service) costs and the prices of a generic PHF (private health facility) [26].

4.2.4 The socio-economic and demographic profile of obese patients

Obese subjects have quite typical socio-demographic characteristics, a profile that public institutions have to recognise to ensure universal access of patients to health care pathways. Most of the subjects are women of childbearing age, or at least young people, more and more often under 35. Many of them have a higher secondary education level or lower, which reflects in a low employment rate and in a medium-low or frankly low income.

\subsubsection{Lack of bariatric anaesthetist}

For patients with a low level of obesity or without serious risk factors the hospitalization in the intensive care unit is a superfluous caution that dilates waiting times. In the absence of a bariatric anaesthetist every patient, regardless of health condition, usually has to wait far more than necessary, until the hospital has a vacant bed in the intensive care unit (because of the priority given to planned and urgent interventions). 


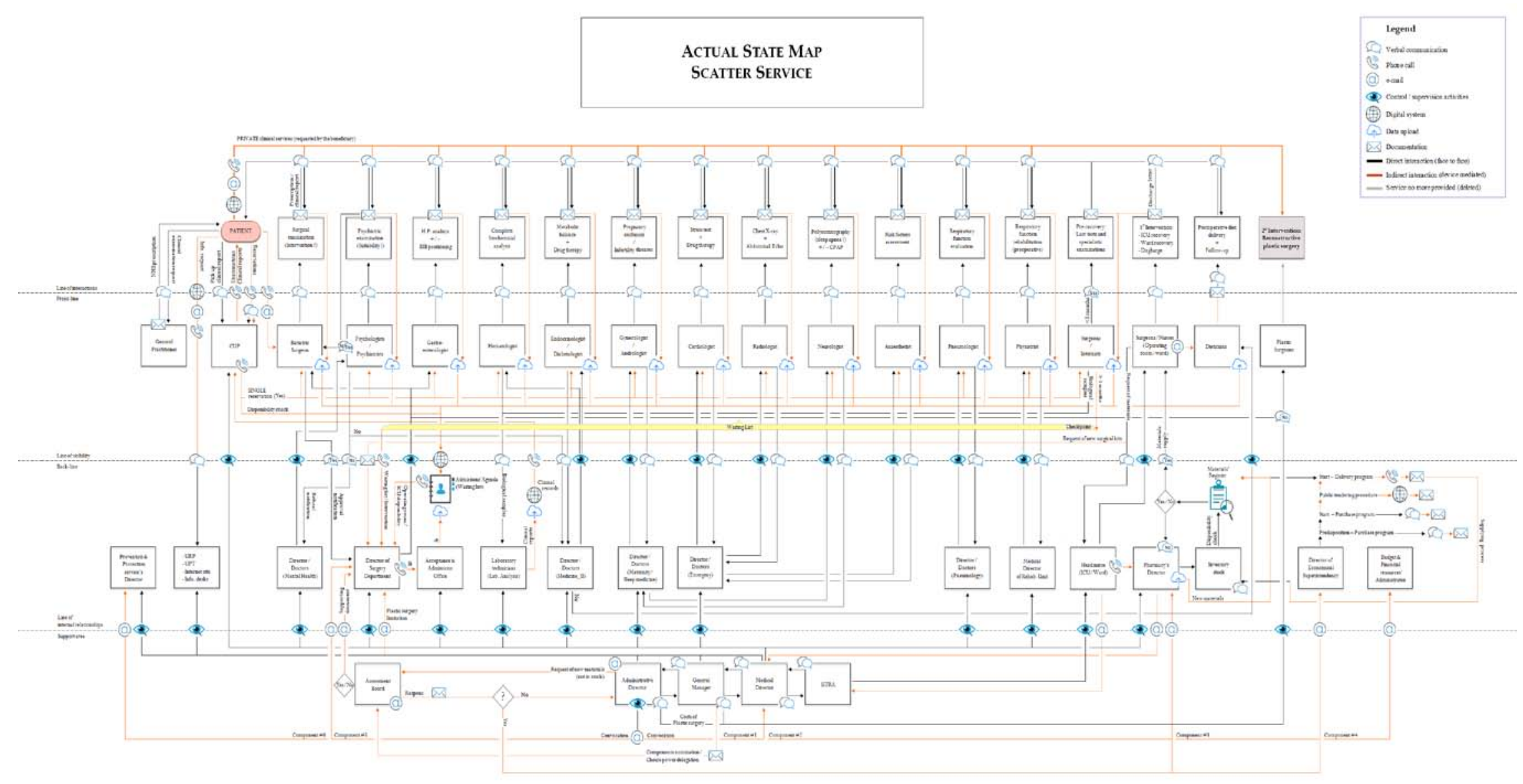

Fig. 1 Actual state map-scatter service. 
PRIVATE clinical services (requested by the beneficiary)

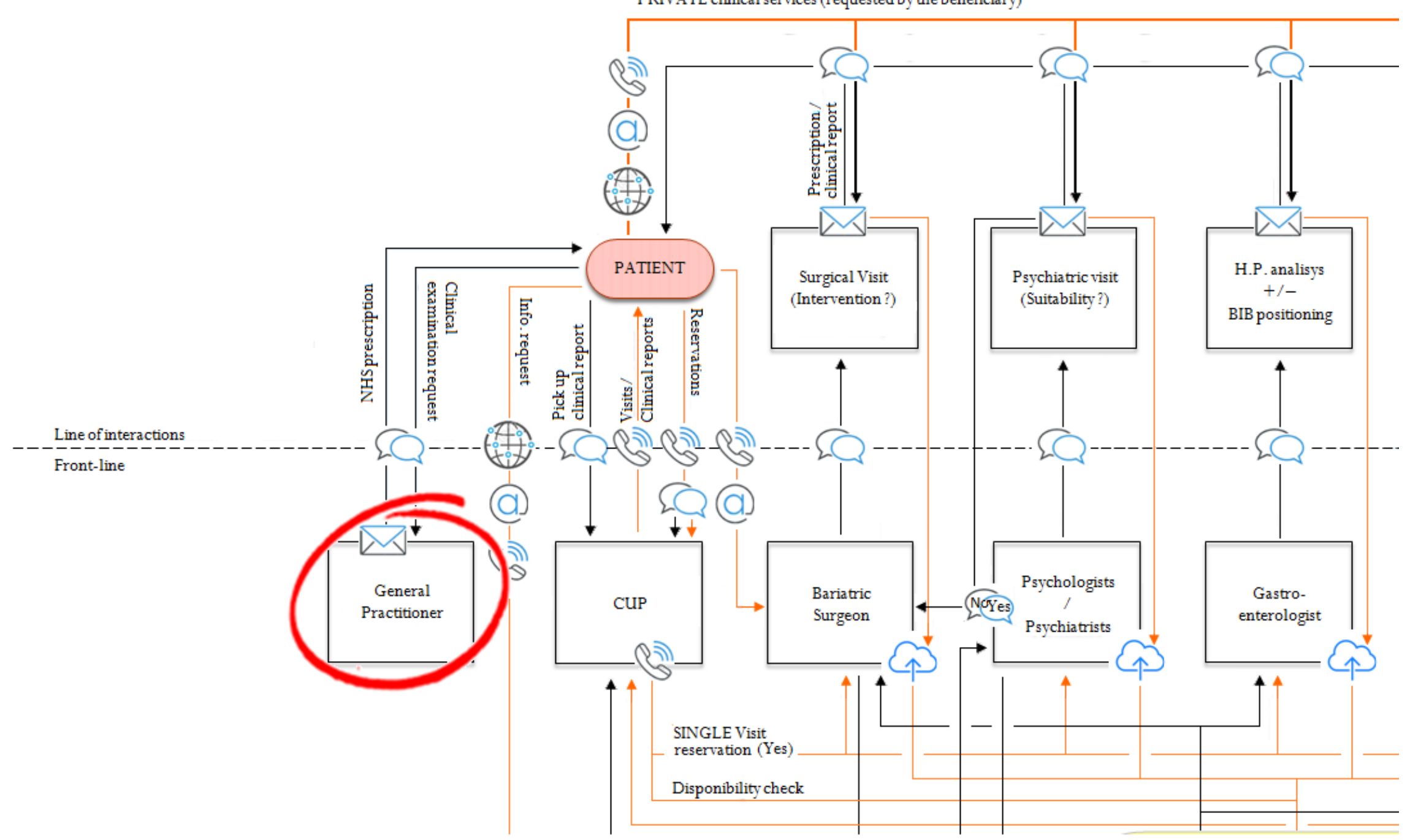

Fig. 2 Section of the actual state map-role of the general practitioner 
Table 1 Average health care expenditure of bariatric surgery patients.

\begin{tabular}{lll}
\hline Visits/Tests & NHS Costs (in $€$ ) & PHF Costs (in €) \\
\hline Biochemical tests (blood, urinary, faecal) & 313.97 & $260-310$ \\
Psychiatric examinations & 38.74 & $130-150$ \\
Surgery examinations & 20.66 & $100-120$ \\
Targeted psychological questionnaire & 56.81 & $70-80$ \\
Specialistic examinations* & $20.66(10)=206.60$ & $100-120(10)=1,000-1,200$ \\
Gastroscopy + biopsy (Helicobacter Pylori) & 99.40 & $250-350$ \\
Electrocardiogram (with stress test) & 36.15 & $50-70$ \\
Andrological or gynecological echography & 51.65 & $70-100$ \\
Chest X-ray & 18.36 & $25-35$ \\
Complete abdominal echography & 60.43 & $70-100$ \\
Spirometry & 37.18 & $60-70$ \\
Polysomnography & 139.44 & $200-220$ \\
Perioperative therapy (drugs and food supplements**) & 150.00 & $180-200$ \\
Bariatric intervention & - & $1,200-7,000$ \\
TOTAL (euro) & $1,231.39 €$ & $3,665-10,015 €$ \\
\hline
\end{tabular}

* Required visits (maximum ten): diabetic, endocrinological, dietetic, andrological or gynecological, hematologic, cardiological, neurological, physiatric, pneumological, and radiological examinations; ** Routine drugs and dietary supplements prescribed: levothyroxine, gastroprotector, antacid drug, vitamin D and vitamin B complex, iron, folic acid, essential amino acid supplements, concentrated omega-3 fatty acids (EPA/DHA), combined multivitamin and multi-minerals supplements.

\subsubsection{Lack of specialized peri-operative personnel}

The lack of nurses specialized in mini-invasive techniques doesn't allow to optimize the intervention time and causes waste of surgical instruments, constant drawn on the inventory stocks, and new orders of materials. In postoperative phase, professionals are often unable to provide adequate assistance to the patients. The methylene blue transit should be performed the day after surgery for an early discharge, but normally the radiologist takes care of this activity only once a week, a gap that obviously originates from the lack of knowledge rather than a real staff shortage.

\subsubsection{The dietician is contacted too late in the process}

The nutritional evaluation of patients and setting of the postoperative diet take place at the end of the surgical path. The request is sent to the dieticians by e-mail and this represents a huge disadvantage in welfare terms. The lack of nutritional intervention in the preoperative period generates difficulties in the management of the patients during the surgical procedure, affects the early restoration of health state and the efficacy of drug therapy. Diets are generally delivered to patients with two or more days of delay, thus compromising the quality of physical recovery during the first 48-72 postoperative hours. Dieticians' ward commitments and the little prior notice for the evaluation of bariatric patients, make it impossible to take care of each case. So, the diets provided are poorly personalized and frequently incoherent with the type of surgical intervention performed (i.e.: excessive amount of food/liquids for patients undergoing gastrectomy).

\subsubsection{Patients' dissatisfaction}

Patients' critics, towards healthcare professionals can be summarized as follows:

- Unclear and poorly personalized diets;

- Absence of instructions in case of feeding difficulties;

- Foods incompatible with allergies and intolerances declared;

- Excessive portions of food/liquids in relation to post-operative digestive possibilities;

- Inadequate support for the management of gastrointestinal symptoms;

- No information about cooking methods;

- Superficial or incomplete follow-up examination; 
- Perception of a low level of interest of the dieticians during the examination;

- Impossibility of comparison with the same dietician at subsequent medical check-ups;

- Discordance between surgeon and dieticians' instructions about dietary supplements;

- Physical activity not specified (in type, timing or intensity).

The poor attention of dieticians to patients' needs determines low motivation levels and complete indifference of subjects about nutritional therapy.

\subsubsection{Inefficient supply process}

Purchases are often fragmentary, and that causes a large amount of bookings (spot orders, no contracts, etc.). The absence of a complete programming of the supply system, which takes to account the needs of beneficiaries and healthcare professionals, make it impossible to meet clinicians' requests in short times.

\subsubsection{Limitation of plastic surgery interventions}

In the three-year period 2015-2017 regional councils have enacted many questionable acts. The most blatant examples are the reduction of reimbursement for bariatric surgery DRGs' and obligation to use DRG 288-economically less profitable than the previous 292-which does not consider complications or comorbidities. This variation distorts the remuneration system, especially of public healthcare facilities, which are currently unable to provide reconstructive plastic surgery interventions to their patients. Actual DRGs do not cover the costs of intervention and hospitalization: all bariatric operation - from the simplest to the most complex-are reimbursed by the National Health Service to the same extent, despite each intervention involves different costs (in terms of occupation of the operating room, materials consumed, days of hospitalization, etc.).

\section{The New Delivery System of the Bariatric Surgery Service}

\subsection{Desirable State Map}

The desirable state map shows the most macroscopic changes, mainly due to the reorganization of work and to the repositioning of healthcare professionals along the bariatric path (Fig. 3). Starting from the critical issues previously discussed, we have developed the desirable state map (or clover service $\left.\operatorname{map}^{7}\right)$.

\subsection{Beneficiaries’ Area and Front-line Zone}

The first examination-conducted by the surgeon and the dietician together-aims to identify the subjects eligible for bariatric surgery (Fig. 4). On the base of anamnestic and anthropometric data (weight, height, BMI, body circumferences and fat mass/lean mass ratios), clinicians will explain the patient what intervention is more adapt to his/her case. Afterwards, the patient will meet the psychologist, who will verify the subject's psychiatric integrity and suitability for bariatric intervention. Submitting to the hospital's CUP service the general practitioner's prescription the patient will book all multidisciplinary examinations needed.

On the same day, the patient will undergo to gynecological (or andrological) examination, gastroscopy and biopsy, in order to exclude pregnancy/reproductive disorders, to examine the gastrointestinal tract configuration and check for any infection from Helicobacter Pylori.

The third examination evaluates the intraoperative risk and will be performed by the cardiologist, the radiologist, and by the anaesthetist, in order to determine which cases need assistance in the intensive care unit.

The fourth clinical examination aims to plan preoperative rehabilitation, if the patient has organic and/or systemic defects.

The last examination corresponds to pre-recovery Here, the internists, the bariatric surgeon, and the anaesthetist will perform the last tests before intervention, which will take place within a week.

\footnotetext{
7 The title "Clover Service" has been assigned to the desirable state map, in light of the structure taken by the service after the placement of the multidisciplinary examinations.
} 


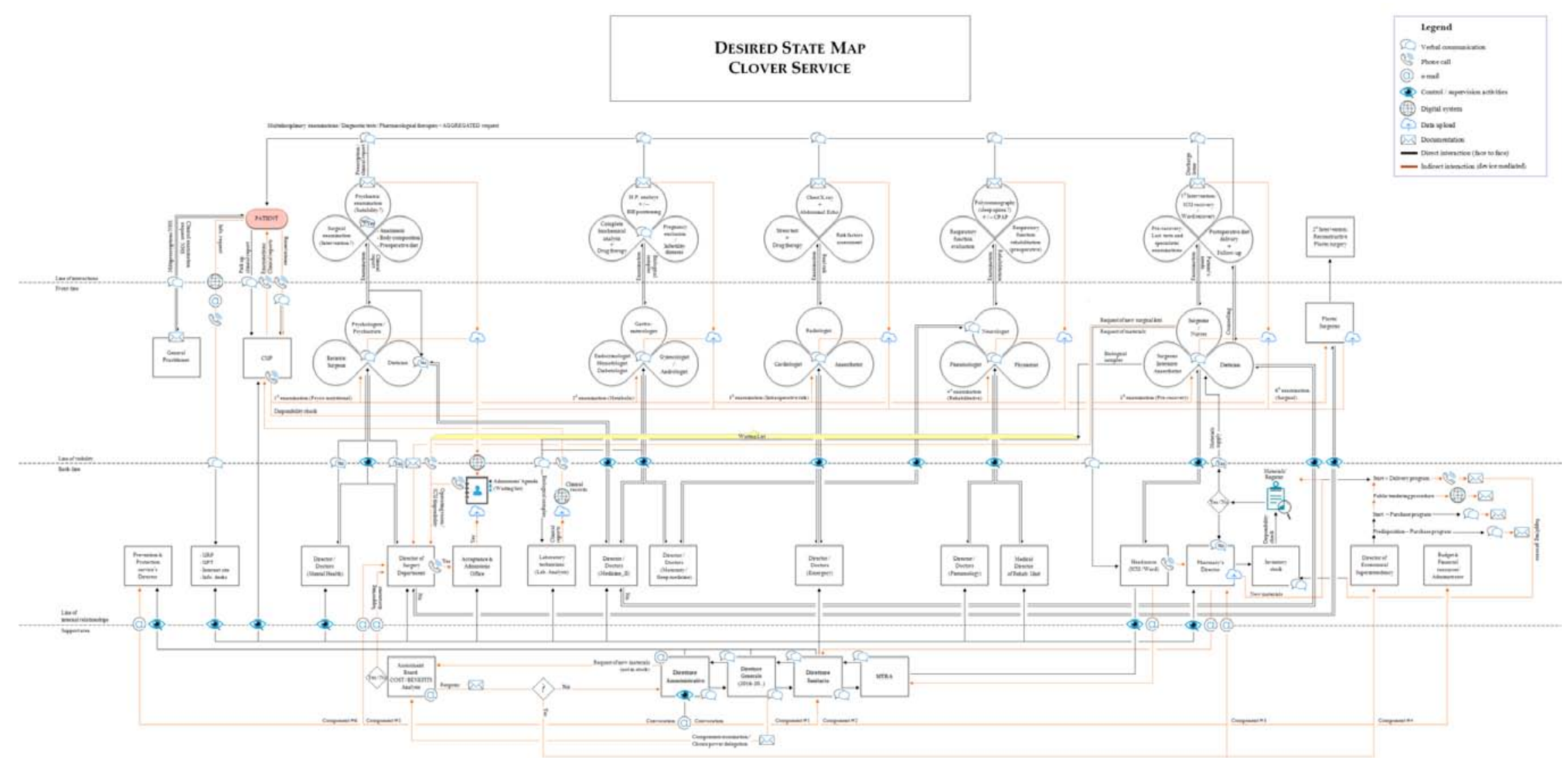

Fig. 3 Desired state map. 
Multidisciplinary examinations/Diagnostic tests/Pharmacological therapies-AGGREGATED request

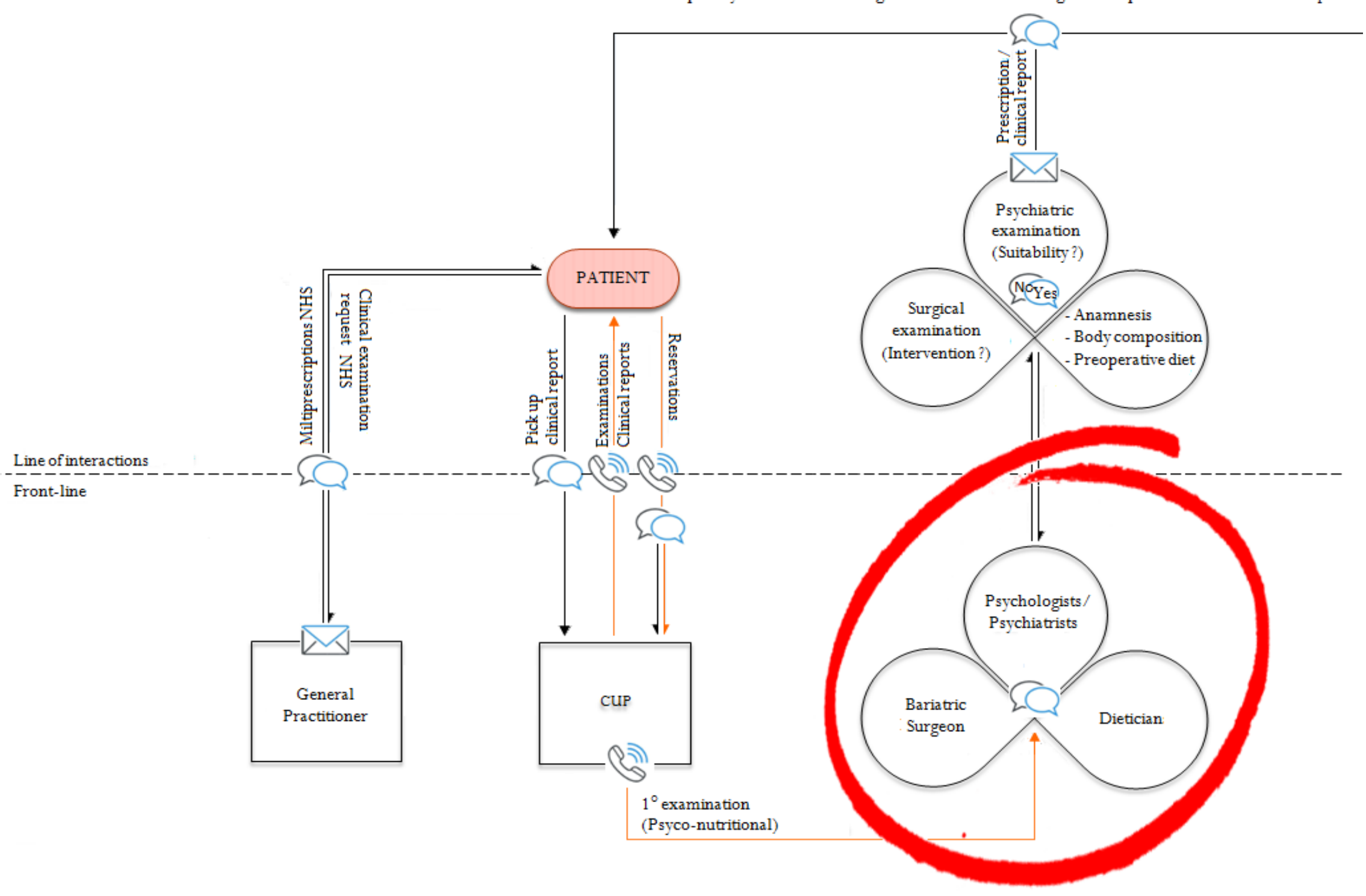

Fig. 4 Section of the desired state map-psyco-nutritional examination. 


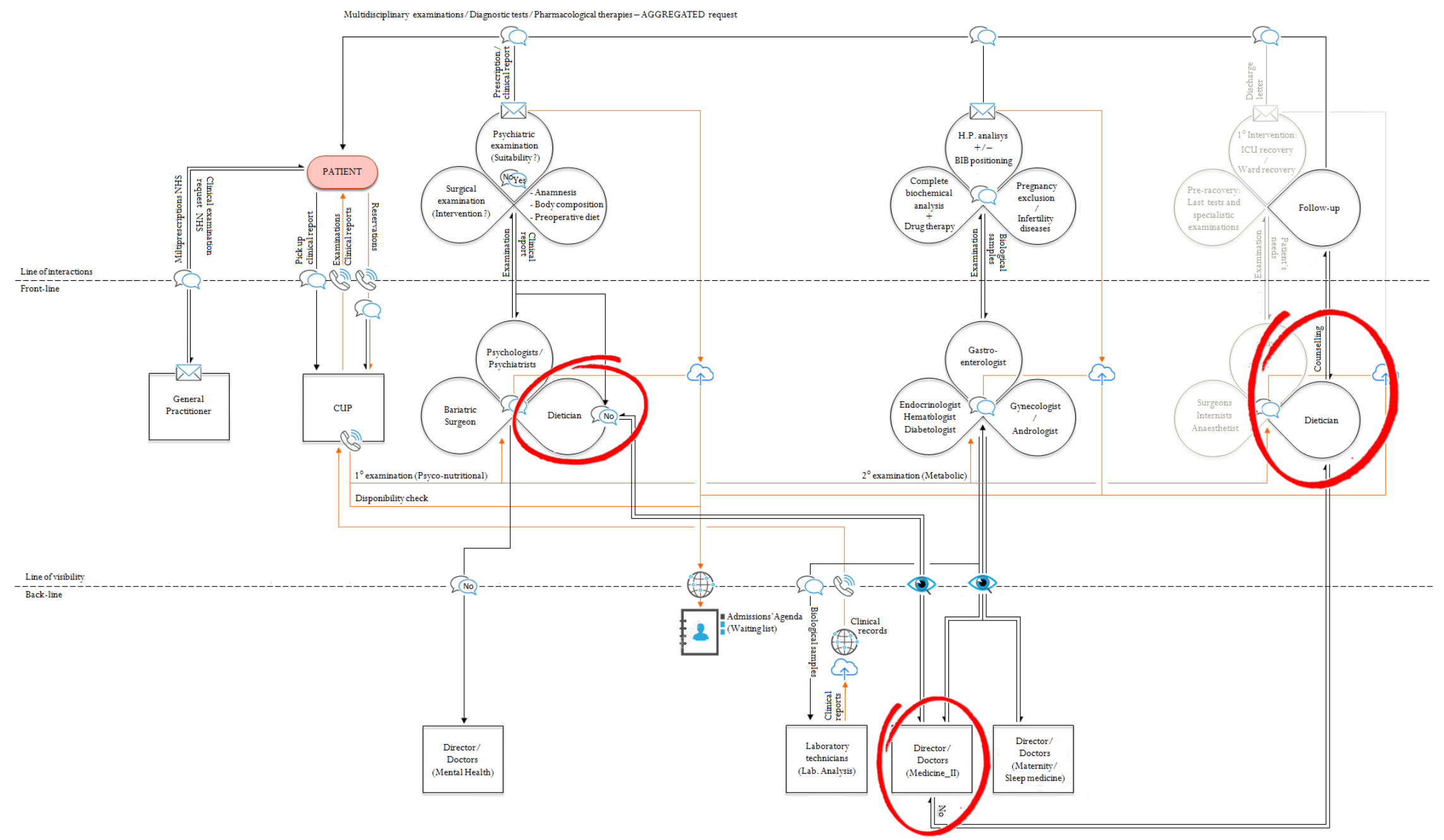

Fig. 5 Section of the desired state map-repositioning of dieticians. 


\subsection{The Support Area of the Bariatric Surgery Service}

Fig. 3 makes it clear that clover structure responds perfectly to the need of harmonization of the bariatric surgery service. The coherent grouping of healthcare professionals-following a logic of professional affinity and departmental affiliation -allows the integration of activities and the aggregation of information. Thus, the direct communication between team members is guaranteed at every stage of the service delivery, creating a direct relationship channel between front-line, back-line, and support area.

\subsection{Micro-organization of Work}

The new bariatric surgery service avoids to the patients all that multiple accesses to the hospital, which previously characterized the diagnostic path. This organizational mode, linear and orderly, increases the effectiveness of the service-in terms of quality delivered and perceived-and promotes hospital efficiency. The radical rearrangement of work respond to patients' needs and personalizing the health care process; this organizational strategy move away the bariatric service by the idea of "curing the disease", approaching it to the responsibility of "taking care of the patient”.

\subsection{Integration of Activities}

The integration of activities (i.e. communication between department professionals and interaction among operating units) is not a simple exchange of clinical information, but a true sharing of meanings between complementary realities, which contribute to achieve common health goals.

\subsection{The "Clover" Organization}

The clinical paths (surgical and medical) should be managed by all team members, in a multidisciplinary perspective. This optimization greatly reduces waiting times: the over fifteen diagnostic examinations of the old delivery system, are now reduced to only five (or six-in case of plastic surgery intervention), giving the bariatric surgery path a semblance of an integrated service. So, reservations arriving to the CUP will be easily planned, while diagnostic and therapeutic activities will be managed in the full respect of the ward agenda. The lack of an operating unit is advantageous, since it does not require additional management costs, recruitment of other healthcare professionals, and a chief surgeon.

\subsection{Service Simplification and Direct Communication among Professionals}

In the new delivery system the interactions change both in the type and number, and face to face relationships have become predominant. The aggregation of diagnostic examinations creates the need of reciprocal exchange of information between professionals and determines a greater involvement of managers, firstly hindered by the fragmentation of front-line actions. Indirect relationships have been confined to formal or official communications (such as operating room reservation, order of materials, etc.), all official procedures that require medical-legal traceability.

\subsection{Reposition Dieticians in the Bariatric Surgery Service}

The importance of dieticians finds its utmost expression in relation to the treatment of obesity. For an obese patient (especially with metabolic syndrome or other co-morbidities), it is essential to follow a preoperative diet: calories restriction reduces intraoperative risks, allows to achieve the full recovery of health state and avoid the occurrence of lethal complications or disabling outcomes in the immediate postoperative period (Fig. 5). We must remember that if the bariatric surgery is not among the patient's cure opportunities (in case of psychiatric unsuitability), the nutritional approach will be the only solution for the patient. In this case, diet is indispensable to improve the chronic course of the 
pathology; diet is, to all intents and purposes, an important project of "prevention within the cure".

\section{Discussion}

What would change for healthcare professionals of the bariatric surgery service?

\subsection{Innovations for the Patient}

Obese subjects are no more considered as ill patients needing care, and not just as persons (subject with rights and duties), but as a "beneficiary", a resource bearer and co-producer of the service of which himself benefits. Therefore, the beneficiary is an individual with a strong relational value, qualified in expressing opinions and judgments about the services and the level of health care provided.

\subsection{New Instructions for Healthcare Professionals}

The "Clover" organization motivates clinicians' participation and collaboration, promotes innovation, and contributes to reduce conflicts and disservices. This method is a very important tool for all hospitals and Public Health's managers which want to undertake significant cultural modification. This perspective supports clinical programming, develops participation, improves Public Health performances, stimulates conscious use of the services, creates transparency about public administration activities and increases communication.

\subsubsection{General practitioners' duties}

General practitioners must be aware of the importance of a timely and correct diagnosis of obesity. They will have to find the main anthropometric risk indices (weight, height, BMI, and waist circumference) in order to formulate diagnosis of obesity and send the patient to specialized hospitals or National Health Service's clinics. If the first outpatient clinical examination introduces the subject to bariatric surgery intervention, the general practitioner will have to prescribe clinical examinations in aggregate mode (multipath request), so that the CUP can do the same and properly plan all examinations with the various specialists.

\subsubsection{Physicians and surgeons}

Obesity diagnosis must always determine immediate introduction of the subject into specific care paths of the National Health Service. The patient must come to the internist's/dietologist's attention through a regional prescription which certifies the diagnosis of obesity. If the surgeon diagnoses obesity to the subject, the clinician will have to address the patient to a public hospital's specialist, in order to get start of specific diagnostic procedures and therapeutic programs.

\subsubsection{Psychiatrist}

Although the psychiatric eligibility of patients to surgical therapy remains in the hands of the psychiatrist, the opinion of the surgeon and the dietician-in light of their professional knowledge—can provide useful details and important information about the personality and the patient's eating attitudes. This contribution is crucial, since it facilitates the discrimination of unsuitable cases for bariatric surgery.

\subsubsection{Dietician}

The dietician will evaluate each aspect of the patient's nutritional state: preoperative assessment, dietary path supervision, food education, nutritional counselling and follow-up activities.

During the first clinical examination with the surgeon, the dietician will carry out the biometric evaluation of the patient, which will include:

- Weight, height and BMI measurement

- Calculation of body composition

- Anamnesis on the eating habits and patient's lifestyle

- Weekly monitoring with metabolic holter

- Evaluation of food diary and constant nutrition counselling

Nutritional counselling should not be confined to programmed follow-up examinations (every six months or once a year), but should be ideally provided to the patient for a lifetime. The results of surgical treatment (percentage of successes, failures, 
complications, and re-interventions) strongly depend on the patient's adherence to the prescribed nutritional controls. The authenticity of human relationship, as well as trust between the dietician and his/her patient, greatly affects clinical outcomes, so these variables should become an integral part of each clinical path.

\subsubsection{Top management operators}

In service companies, the delegation of decision-making at the lowest levels of the organizational pyramid-to the operators in direct contact with the beneficiary-is crucial to achieve optimal results and the empowerment of the entire service.

Even though recently there have been significant changes in public administration the change of mentality towards the concept of "serve the citizens" has just begun. In the medical field, the top management has always been considered as the "supporting pillar", which is totally unreasonable if we think that in the industrial culture no one would ever consider as "support" the vertex of an organization (intended as a pyramid) [27]. In the future, hospitals' top management will be increasingly solicited to support clinical activities; they will have to pass from an exclusive supervisory task (a mere enforcement of rules and ex-post controls) to a comparison activity aimed to the sharing of solutions among professionals. This was the ultimate goal of the important programming work of our research group.

6.2.6 Impact on Public Health and healthcare territorial articulations

Although our project has involved a single hospital service, it focuses on the goal of reorganizing and strengthening the territorial network, especially in the area of chronicity and severe obesity (the most important chronic illness of the last decade). This means that all levels of Public Health, from front-line professionals in direct contact with the patient, to the top management of hospitals, ASST $^{8}$, ATS and

8 Azienda Socio-Sanitaria Territoriale: Territorial Socio-Sanitary Company (English). regional institutional bodies, must be informed and likewise they will offer their contribution.

Because of the well known correlation between obesity and poverty, patients with pathological obesity and candidate to bariatric surgery should have a specific exemption. Hospitals' top management will have the responsibility to obtain the approval by the institutional bodies, so that Regions can divulge new directives and provide exemptions, in line with the real health needs of this fragile population.

General practitioners should be the first professional involved in the diagnostic-therapeutic pathway of the obese patient. Therefore, this project needs to be approved by the top managers of different levels of the healthcare service, so that general practitioners can fulfil their tasks. Poor participation of general practitioners, as well as inability to engage them in the very early stages of the bariatric path, underlines the need to re-evaluate the role of these clinicians. The new delivery system, in fact, shows that even the prescriptions of the general practitioners can be conceived as a series of stages, a strategy that does not require changes of the regional/national actual tariff.

\subsubsection{Limitations of the study}

Share and adopt our organizational model would be the best strategy to ensure continuity to the therapeutic pathways and to guarantee access of all obese patients to the Public Health network. However, the study has a great limitation. The lack of knowledge of the method and its complexity (number of documents to be viewed and subjective variables to consider), in fact, limit the application of Service Mapping in the healthcare field. This methodology requires a multi-perspective approach and an adequate technical training. In addition, Service Mapping is highly challenging/onerous in the analysis and an elaboration phases - which represent indispensible steps to design the maps-requires great attention during the representation of work organization: the iconic description must be really meticulous to exactly 
describe the processes. For these reasons the results of our study, although of great potential, can be successfully used only by properly trained professionals. So, we need to invest time, both for the training of health care managers and the definition of Service Mapping's guidelines in healthcare context.

\section{Conclusions}

Thanks to the potential of Service Mapping method, we have developed a new clinical pathway that truly guarantees the centrality of the patient and the involvement of all operators, top management included [28].

The unequivocal conclusion of the studies conducted on obese patients, is that bariatric surgery completely cuts down National Health Service costs within only five years from intervention, a result not otherwise achievable with traditional pharmacological or dietary therapy [29-31]. Bariatric surgery does not simply improve the quality and the lifespan of obese people, but it also allows an unparalleled reduction of the socio-sanitary expenditure, if compared to any other therapeutic approach [32-34]. The success of bariatric therapy is ensured by the presence of a strong interdisciplinary team, able to take care of the patient at all stages of the clinical pathway. It's a proper "way of being" even before a "way of behaving", which allows the application of specialized knowledge and skills to the complexity of cross-sectoral problems, in a systemic vision of the reality.

Through this study, we exhort Public Health's leaders not to forget the importance of the equity of access to healthcare and therapeutic pathways, the support of clinicians in their activities and the recognition of the centrality of the beneficiary. The risk of not understanding the need of a change is, of course, atrophy at every level of Public Health. A service is "a quality service" if all actors involved are respected in their rights and if they actively participate in the production of the service [35]. So, quality should be read as the optimal mediation point, that is, the best degree of integration between different values: expectations, aspirations, needs and rights of people around the service (owners, managers, professionals, direct users and their families) [36].

As professionals directly involved, we hope for an immediate return to the authentic "culture of the service" and we will actively contribute to the adoption of an organizational logic that recognizes the beneficiary's basic needs. In the absence of this, healthcare providers will have to realize that Public Health can no longer be defined as a "network of personal services". Moving on obtusely in this direction, the National Health Service-not only the Italian one, but of any country in the world-will run into a progressive and irreparable disintegration, which will make it impossible to protect the health of the most fragile patients. There will be no chance to save our National Health Service until we have the humility to take a step back, recognizing the centrality of people that we daily intend to cure, assist, and serve.

\section{Acknowledgments}

Contributorship statement: All those designated as Authors have covered a specific role in writing the manuscript and meet the four criteria for authorship, as stated in the ICMJE Recommendations. Lara Loreggian designed the research; Lara Loreggian, Maria Elisa Sartor and Marco Rovati supervised the research and wrote the paper; Laura Strohmenger reviewed the manuscript. Each Author had a specific responsibility for final content. All Authors read and approved the final manuscript.

\section{Competing Interests}

We confirm that there are no known conflicts of interest associated with this work and there has been no financial support that could have influenced its outcome.

Funding: No source of funding has a role in the study's design, conduct, and reporting. 
Data sharing statement: We confirm that we will provide as attachments all figures, tables, information and tools used to perform the study and all documents related to it (Customer satisfaction, Triennial Performance Plan, organizational chart, macro-structure of the Regional Health Service, P. O. A. and official acts).

\section{References}

[1] World Health Organization. 2001. "Obesity: Preventing and Managing the Global Epidemic.” Report of a WHO Consultation (WHO Technical Report Series 894).

[2] International Obesity Taskforce. 2005. "London, United Kingdom: International Obesity Taskforce.” http://www.iotf.org/aboutobesity.asp.

[3] Roux, L., and Donaldson, C. 2004. "Economics and Obesity: Costing the Problem or Evaluating Solutions?” Obes Res. 12 (2): 173-9.

[4] American Obesity Association (AOA). 2005. "Costs of Obesity."

[5] Calle, E. E., Rodriguez, C., Walker-Thurmond, K., and Thun, M. J. 2003. “Overweight, Obesity, and Mortality from Cancer in A Prospectively Studied Cohort of U.S. Adults.” N Engl J Med. 348: 1625-38.

[6] Adams, K. F., Schatzkin, A., Harris, T. B., Kipnis, V., Mouw, T., Ballard-Barbash, R., Hollenbeck, A., and Leitzmann, M. F. 2006. "Overweight, Obesity, and Mortality in a Large Prospective Cohort of Persons 50 to 71 Years Old.” N Engl J Med. 355: 763-78.

[7] Artalejo, F. 2003. "A Simple Estimate of Mortality Attributable to Excess Weight in the European Union." Eur J Clin Nutr. 57: 201-8.

[8] ISTAT. 2016. Risk Factors for Health. Press Release.

[9] PASSI Surveillance System. 2009. Progress of Healthcare Companies in Italy. Survey 2007-2009.

[10] Donia Sofio, A., Meneguzzo, M., Mennini, F. S., and Spandonaro, F. 2007. "Evolution of Health Expenditure in Italy in OECD Countries.” CEIS Health Report.

[11] Brolin, R. E. 2002. "Bariatric Surgery and Long-term Control of Morbid Obesity.” JAMA 288: 2793-6.

[12] Clegg, A., Colquitt, J., Sidhu, M., Royle, P., and Walker, A. 2003. "Clinical and Cost Effectiveness of Surgery for Morbid Obesity: A Systematic Review and Economic Evaluation.” Int J Obes Relat Metab Disord 27: 1167-77.

[13] SICOB (Italian Society of Obesity Surgery and Metabolic Diseases). 2012. "Guidelines and State of the Art of Bariatric and Metabolic Surgery in Italy.” EDISES, Naples.

[14] Sant'Anna High School. 2009. Systematic Review of International Studies on the Social Cost of Obesity. Pisa.
[15] Morino, M., Toppino, M., Forestieri, P., Angrisani, L., Allaix, M. E., and Scopinaro, N. 2007. "Mortality after Bariatric Surgery: Analysis of 13,871 Morbidly Obese Patients from a National Registry.” Ann Surg. 246: 1002-9.

[16] Inelmen, E. M., Toffanello, E. D., Enzi, G., Gasparini, G., Miotto, F., Sergi, G., and Busetto, L. 2005. "Predictors of Drop-out in Overweight and Obese Outpatients." Int $J$ Obes. 29: 122-8.

[17] Angrisani, L., Lorenzo, M., and Borrelli, V. 2007. "Laparoscopic Adjustable Gastric Banding Versus Roux-En-Y Gastric Bypass: 5-year Results of a Prospective Randomized Trial.” Surg Obes Relat Dis. 3: 127-33.

[18] Bitner, M. J., Ostrom, A. L., and Morgan, F. N. 2008. "Service Blueprinting: A Practical Technique for Service Innovation.” California Management Review 50 (3): 66-94.

[19] Kingman-Brundage, J. 1989. “The ABC’s of Service System Blueprinting: Designing a Winning Service strategy.” In Proceedings of 7th Annual Services Marketing Conference. American Marketing Association. Chicago, 30-3.

[20] Bate, P., and Robert, G. 2006. "Experience-based Design: from Redesigning the System around the Patient to Co-designing Services with the Patient.” Qual Saf Health Care 15: 307-10.

[21] Berry, L. L., Carbone, L. P., and Haeckel, S. H. 2002. "Managing the Total Customer Experience.” USA MIT Sloan Management Review, 85-89.

[22] Kingman-Brundage, J. 1995. Service Mapping: Back to Basics. Chichester, John Wiley \& Sons.

[23] Hewing, M. 2014. Business Process Blueprinting: A Method for Customer Oriented Business Process Modeling. Wiesbaden.

[24] Paquet, C., St-Arnaud-Mckenzie, D., Ferland, G., and Dubé, L. 2003. “A Blueprint-based Case Study Analysis of Nutrition Services Provided in a Midterm Care Facility for the Elderly.” J. Am Diet Assoc. 103 (3): 363-8.

[25] Polaine, A., Løvlie, L., and Reason, B. 2013. "Service Design: From Insight to Implementation.” Brooklyn, N.Y. Rosenfeld Media.

[26] Health Minister. 2012. Tariff Nomenclature of Specialist Ambulatory Health Care.

[27] Carlzon, J. 1989. “Moments of Truth.” Harper Business, 15.

[28] Shostack, L. G. 1977. "Breaking Free from Product Marketing.” Journal of Marketing 41: 76-9.

[29] Raebel, M. A., Malone, D. C., Conner, D. A., Xu, S., Porter, J. A., and Lanty, F. A. 2004. "Health Services Use and Health Care Costs of Obese and Nonobese Individuals.” Arch Intern Med 164: 2135-40. 
[30] Van Mastrigt, G. A., van Dielen, F. M., Severens, J. L., Voss, G. B., and Greve, J. W. 2006. "One-year Cost-effectiveness of Surgical Treatment of Morbid Obesity: Vertical Banded Gastroplasty versus Lap-band.” Obes Surg. 16: 75-84.

[31] SICOB. 2015. "Obesity, A Pandemic: Innovations of Surgical Therapy.” In Proceedings of SICOB Conference, Rome.

[32] Karlsson, J., Taft, C., Ryden, A., Sjostrom, L., and Sullivan, M. 2007. “Ten-year Trends in Health-related Quality of Life Ater Surgical and Conventional Treatment of Severe Obesity: The SOS Intervention Study.” Int $J$
Obes 31: 1248-61.

[33] World Health Organization. 2006. "The Challenge of Obesity in the WHO European Region and the Strategies for Response.” In Proceedings of Ministerial Conference. Istambul.

[34] International Obesity Task Force. 2007. London.

[35] Fließ, S., and Kleinaltenkamp, M. 2004. "Blueprinting the Service Company: Managing Service Processes Efficiently.” Journal of Business Research 57 (4): 392-404.

[36] Shostack, L. G. 1984. "Designing Services that Deliver. Harvard Businerr Review.” Gen-Feb 61: 133-9. 